\title{
Nachruf auf den Heimatforscher Manfred Ruttkowski
}

(1932-2015)

\author{
Volkmar Geupel
}

Am 26. März 2015 verstarb in Dresden 82-jährig Manfred Ruttkowski, der sich für die Wiedereinführung der historischen Wegezeichen in der Dresdener Heide und später bei der Erfassung und Bestimmung der denkmalrelevanten Flächen von Altstraßen im westlichen und mittleren Erzgebirge große Verdienste erworben hat. Sein heimatgeschichtliches Engagement fällt in seine zweite Lebenshälfte, als er nach einer Krebserkrankung Mitte der 1980er Jahre seinen Ingenieurberuf zunächst nur noch eingeschränkt ausüben konnte und 1990 sogar ganz aufgeben musste.

Manfred Ruttkowski wurde am 26. Juli 1932 in Stadtilm im heutigen Ilmkreis in Thüringen geboren. Nach dem Schulbesuch, der ihn aus politischen Gründen nicht bis zum Abitur geführt hatte, erlernte er den Beruf eines Werkzeugmachers. Als bester Lehrling seines Jahrganges wurde er 1954 an die Fachschule für Kraft- und Arbeitsmaschinenbau in Meißen delegiert, und von 1955 bis 1958 besuchte er die Ingenieurschule für Flug-

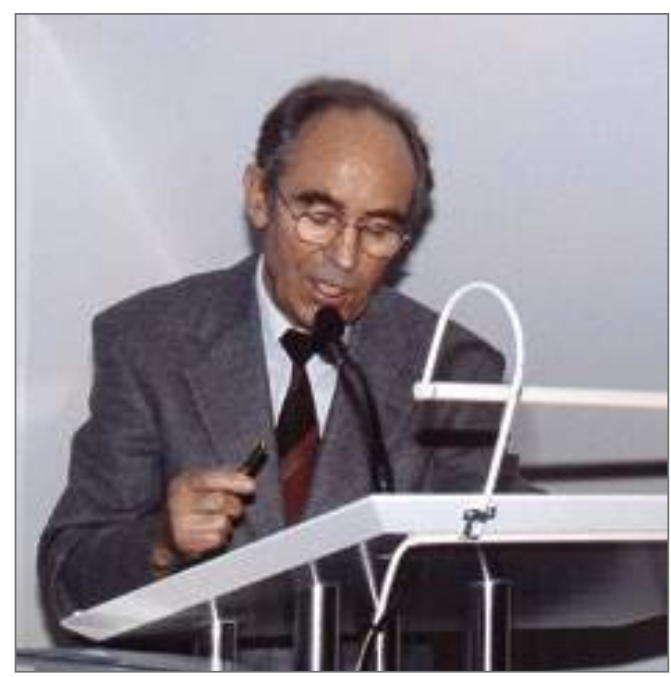

zeugbau in Dresden. Nach der Tätigkeit in Betrieben der Rundfunk- und Fernsehempfangstechnik in Radeberg, Dresden und Dresden-Gittersee war Manfred Ruttkowski ab 1980 als Abteilungsleiter und Hauptkonstrukteur im Zentrum für Wissenschaft und Technik Dresden beschäftigt.

Seine außerberuflichen Interessen galten erst technischen und naturwissenschaftlichen Disziplinen wie der Optik, Fotografie und Astronomie; er baute eine Camera obscura und ein Fernrohr, mittels dessen er Himmelskörper fotografieren konnte. Die Beobachtung der Sterne führte Manfred Ruttkowski in die Astronomische Gesellschaft, deren Mitglied er wurde. Nach seiner schweren Erkrankung wandte er sich dem regionalgeschichtlichen Problem der historischen Wegezeichen in dem großen zusammenhängenden Waldgebiet im Norden Dresdens, der Dresdener Heide, zu. Er schloss sich der Interessengemeinschaft Dresdener Heide an und setzte sich für die Wiedereinführung der roten Heidezeichen zur Wiederbelebung einer jahrhundertealten kulturhistorischen Tradition in einer Ratsvorlage des Stadtrates ein. 1987 erschien zu der Problematik der historischen Wege und Wegezeichen der Dresdener Heide eine von ihm verfasste Studie. ${ }^{1}$

Als nach der Wiedervereinigung Deutschlands die ehemaligen Länder wieder hergestellt worden waren, wurde das Landesmuseum für Vorgeschichte Dresden, das in der DDR mit der Wahrnehmung der Bodendenkmalpflege in den drei sächsischen Bezirken Dresden, Karl-Marx-Stadt/Chemnitz und Leipzig beauftragt war, in das Landesamt für Archäologie Sachsen umgewandelt. Zu einer vorrangigen Aufgabe der Archäologischen Denkmalpflege wurde auf Grund des zu er-
1 Manfred Ruttkowski: Historische Zeichen und Wege der Dresdener Heide. Ein Beitrag zur Heimatgeschichte, hrsg. vom Kulturbund der DDR, Dresden 1987.

2 Deren Schutz regelt seit 1993 das die DDR-Verordnung zum Schutze und zur Erhaltung der ur- und frühgeschichtlichen Bodenaltertümer vom 28. Mai 1954 ablösende Gesetz zum Schutz und zur Pflege der Kulturdenkmale im Freistaat Sachsen vom 3. März 1993, geändert 4. Juli 1994, kurz Sächsisches Denkmalschutzgesetz (SächsDSchG) genannt.

Manfred Ruttkowski bei einem Vortrag zum Tag der Landesarchäologie am 25. November 2000 Foto: Landesamt für Archäologie 
Hohle des Altstraßenzuges Altenburg - Kühberg - Preßnitzl Přisečnice im "Stockholz" zwischen Elterlein und Schlettau Foto: K. Geupel, 2015 wartenden Aufbaues in den Städten und Dörfern und des Ausbaues der Verkehrswege eine Schnellerfassung der archäologischen Kulturdenkmale ${ }^{2}$ in einer gegenüber dem alt geschützten Bestand an Bodendenkmalen meist Einzeldenkmalen - erheblich erweiterten Form. In diesem Zusammenhang erhielt Manfred Ruttkowski zu Beginn des Jahres 1992 eine befristete Teilzeit-Anstellung als Mitarbeiter für die Denkmalsinventarisation und wurde von der Amtsleitung dem Gebietsreferat Regierungsbezirk Chemnitz zugeordnet. In den erzgebirgischen Kreisen, wo auf Grund der geomorphologischen Bedingungen und des vergleichsweise hohen Waldanteils Altstraßenrelikte über längere zusammenhängende Strecken erhalten geblieben sind, bildeten diese einen wesentlich größeren Teil der inventarisierten Denkmale als in den Kreisen des sächsischen Niederlandes. Als seinerzeitiger Refe-

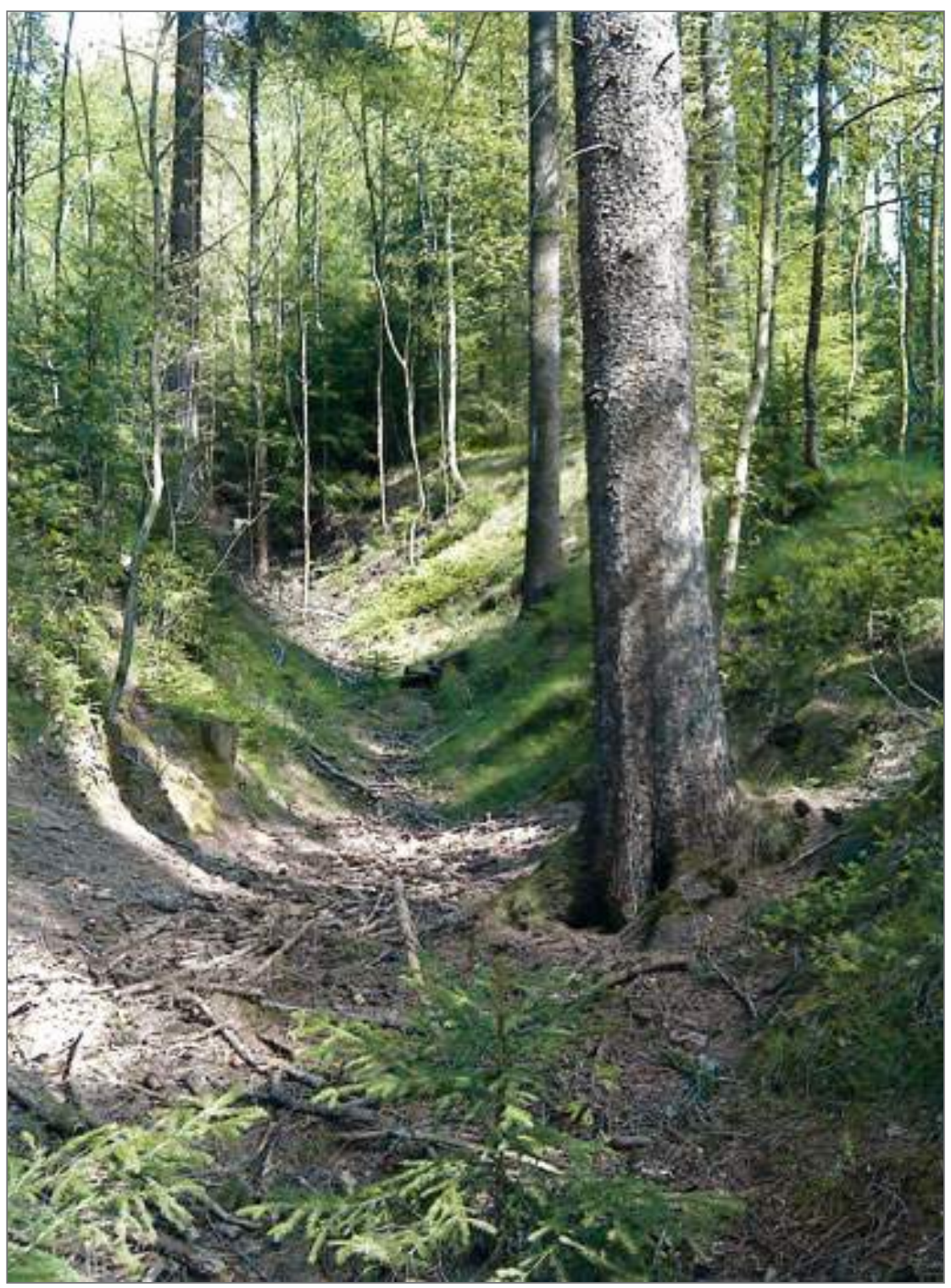

ratsleiter für den Regierungsbezirk Chemnitz schlug ich deshalb Manfred Ruttkowski vor, sich in einem Anschlussprogramm der ältesten gebirgsüberschreitenden Verkehrszüge, der Böhmischen Steige und der ihren Trassen folgenden hochmittelalterlichen Fernstraßen im mittleren und westlichen Erzgebirge anzunehmen und nach den Prinzipien der Denkmalsinventarisation die Altstraßenverläufe auf der Topographischen Karte TK 10 (1 : 10 000) struktur- und flächengerecht zu erfassen. Ziel sollte die flächenhafte Ausweisung der Altstraßenrelikte sein, die als archäologische Denkmale (= Bodendenkmale) schutzfähig im Sinne des Sächsischen Denkmalschutzgesetzes von 1993 sind.

Förderlich für das Vorhaben war, dass die Altstraßenproblematik des Erzgebirges in mehreren, am Fachbereich Alte Geschichte der Pädagogischen Hochschule Dresden unter der Ägide von Prof. Dr. Gerhard Billig (geb. 1927) entstandenen Diplom- und Magisterarbeiten, vor allem aber in der (leider unveröffentlichten) Dissertation von Renate Wißuwa, wissenschaftlich aufgearbeitet vorlag. Dem spezifischen Kriterium der Denkmalsinventarisierung, nämlich die struktur- und flächengerechte Aufnahme der historischen Straßenverläufe zwecks Ausweisung von Denkmalen, konnte die punktuelle oder lineare Darstellung auf dafür ungeeigneten (seinerzeit aber ausschließlich zur Verfügung stehenden) Karten in den genannten Arbeiten nicht genügen und machten eigene Geländebegehungen und die Erfassung der denkmalrelevanten Bereiche auf der Topographischen Karte TK 10 unumgänglich, wofür diese Forschungsarbeiten jedoch eine unverzichtbare Grundlage bildeten. Mit der ihm eigenen akribischen Arbeitsweise hat Manfred Ruttkowski die Exkursionen ins Gelände mit der Auswertung der Literatur und des Kartenmaterials am Schreibtisch vorbereitet. Die Geländebegehungen erfolgte stets zu zweit, was einer gemeinsamen Befundanalyse dienlich war und das beiderseitige Abschreiten von Hohlensträngen ermöglichte, wobei Straßenbreiten und -längen sowie die Tiefen der Hohlen eingeschätzt und auf dem Kartenblatt notiert wurden. Zu seinen abschließenden Befundund Arbeitsberichten gehören Ausschnitte der TK 10, des Messtischblattes (1: 25 000) und der TK 25 (für die Digitalisierung), des sog. Meilenblattes (1:12 000) und, sofern vorhanden, von Flurkarten (1: 5 000). 


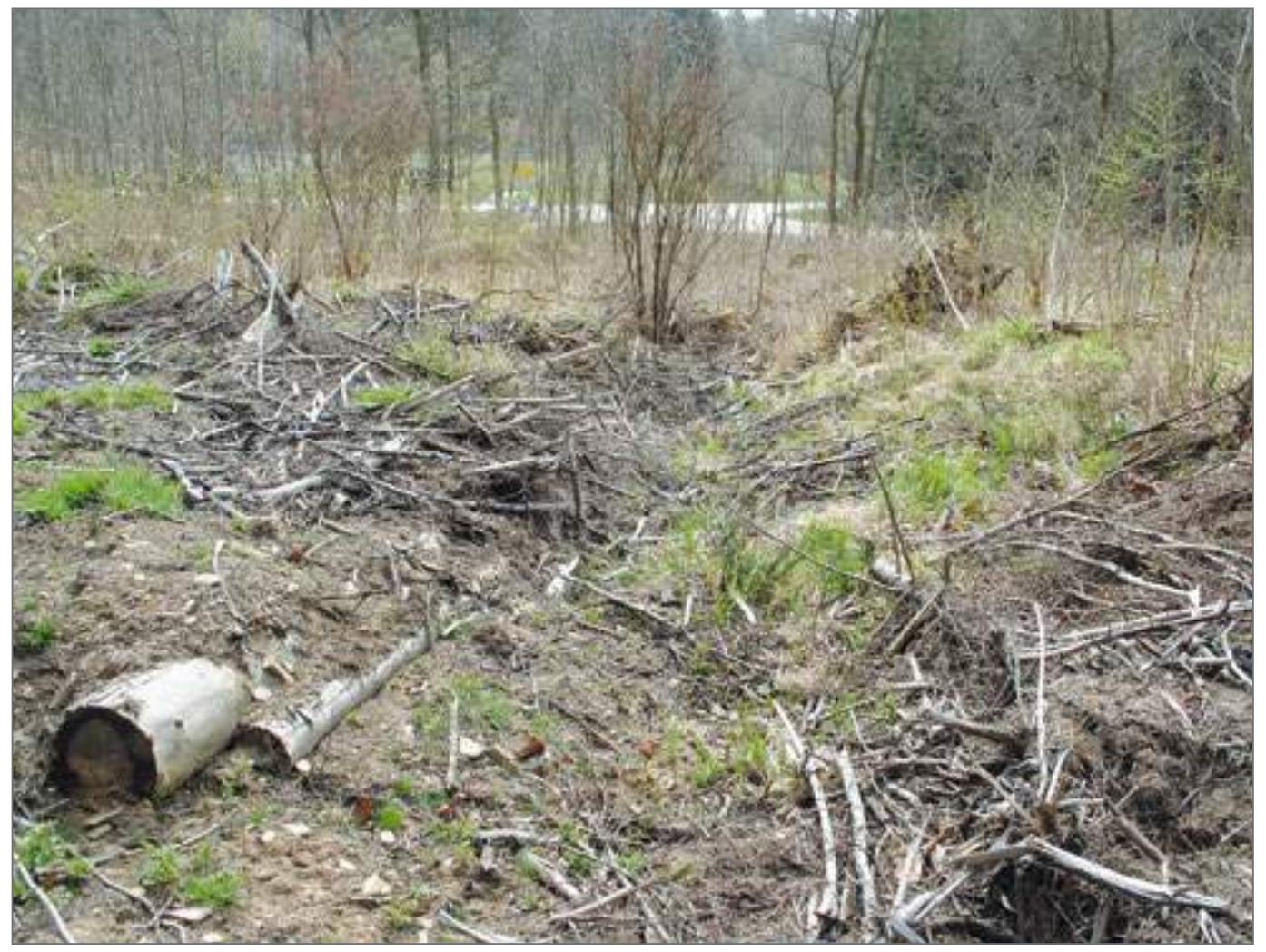

Sein Projekt schloss Manfred Ruttkowski mit einem Aufsatz ab, der fraglos nicht nur eine wissenschaftliche, sondern für das methodische Vorgehen bei vergleichbaren Altstraßenaufnahmen beispielhafte Arbeit darstellt. ${ }^{3}$ Die aufgenommenen Geländebefunde seines in 25 Arbeitsfelder eingeteilten Arbeitsgebietes erfahren dort eine präzise Kartierung und in einem resümierenden Katalog die gedrängte kommentierende Auswertung. Die intensive Beschäftigung mit historischen Karten, wie beispielsweise mit der des Geyerschen Waldes von Balthasar Zimmermann aus dem Jahre 1616, führte Manfred Ruttkowski zum Neufund einer auf 4,5 km Länge den Wald um die Felsformation des Schatzensteins mit zwei Linien querenden Altstraße. ${ }^{4}$ Diese scheint einen älteren Steig von Altenburg über Glauchau - Elterlein -Schlettau nach Kühberg anzuzeigen, aus welchem durch Steigdynamik die Straßen Altenburg Glauchau - Zwönitz - Geyer nach Satzung und Altenburg - Zwickau - Grünhain - Elterlein - Schlettau nach Kühberg hervorgingen, wobei die verbindende Querung des Waldes um den Schatzenstein aufgelassen wurde.

Manfred Ruttkowski hat den mit seiner Anstellung am Landesamt für Archäologie verbundenen Auftrag hervorragend erfüllt. Die von ihm erfassten und dokumentierten Altstraßenrelikte des westlichen und mitt- leren Erzgebirges sind als archäologische Denkmale registriert und unterliegen den Schutzbestimmungen des Sächsischen Denkmalschutzgesetzes. Damit aber die zu Denkmalen erklärten Bereiche von den Besitzern und Nutzern des Geländes, auf denen sie liegen, respektiert und als solche behandelt werden, bedarf es einer zielgerichteten Aufklärung, insbesondere der Forstwirtschaftsbetriebe als häufigste Eigentümer von Flächen mit Altstraßendenkmalen, entsorgen diese doch den bei der "Holzernte“ anfallenden Abfall geradezu regelhaft in den Hohlen oder überfahren und zerstören diese mit ihren schweren Rück- und Zugmaschinen.

Manfred Ruttkowski war ein bescheiden auftretender, mit großem Fleiß und Freude an der Sache arbeitender Kollege. Gern erinnere ich mich an die mit der Altstraßenaufnahme verbundenen Geländebegehungen, bei welchen ich ihn in der Regel als der genannte zweite Mann begleiten konnte, und die dabei geführten fachlichen Diskussionen.

Das heimatgeschichtliche Engagement Manfred Ruttkowskis fand mit der Überreichung der Ehrenurkunde des Sächsischen Landespreises für Heimatforschung durch Frau Staatsministerin Brunhilde Kurth (geb. 1954) 2012 eine verdiente Anerkennung.
Mit Forstabfall verfüllte Hohle des Altstraßenzuges Altenburg Remse - Reitzenhain - Komotau/ Chomutov am "Alten Gericht" bei Marienberg Foto: Volkmar Geupel, 2015

\section{Autor}

Volkmar Geupel

Dresden 